\title{
Intuitionistic Fuzzy Pseudo-Boolean Implicative Filters of Lattice Pseudo-Wajsberg Algebras
}

\section{A. Ibrahim, K. Jeya Lekshmi}

\begin{abstract}
In this paper, we introduce the notion of an intuitionistic fuzzy pseudo-Boolean implicative filter of lattice pseudo-Wajsberg algebra (LPWA) and to investigate some properties with illustrations.
\end{abstract}

Keywords: Pseudo-Boolean implicative filter; Fuzzy pseudo-Boolean implicative filter; Intuitionistic Fuzzy pseudo-Boolean implicative filter; Lattice pseudo-Wajsberg algebra(LPWA)

\section{INTRODUCTION}

Pseudo-Wajsberg algebras were introduced by Ceterchi Rodica [2]. Pseudo-Wajsberg algebras are generalizations of Wajsberg algebras. Boolean algebra is the branch of algebra in which the values of the variables are the truth values true and false. Recently, the authors introduce the definition of pseudo-Boolean and fuzzy pseudo-Boolean implicative filter of lattice pseudo-Wajsberg algebra and obtain some related properties. The aim of this paper is to introduce the definition of an intuitionistic Fuzzy pseudo-Boolean implicative filter of LPWA and obtain some properties.

\section{PRELIMINARIES}

In this section, we recall some basic definitions and their properties which are helpful to develop the main results.

Definition 2.1[2]. An algebra $(\mathcal{A}, \rightarrow, \sim,-, \sim 1)$ is called a LPWA if it satisfies the following axioms for all $x, y \in \mathcal{A}$,

(i) A partial ordering " $\leq$ " on a LPWA $\mathcal{A}$, such that

$$
\text { (iii) } \begin{aligned}
x \wedge y & =\left(x \sim(x \rightarrow y)^{\sim}\right)^{-}=\left((x \rightarrow y) \rightarrow x^{-}\right)^{\sim} \\
& =\left(y \sim(y \rightarrow x)^{\sim}\right)^{-}=\left((y \rightarrow x) \rightarrow y^{-}\right)^{\sim} \\
& =\left(y \rightarrow(y \sim x)^{-}\right)^{\sim}=\left((y \sim x) \sim y^{\sim}\right)^{-} \\
& =\left(x \rightarrow(x \sim y)^{-}\right)^{\sim}=\left((x \sim y) \sim x^{\sim}\right)^{-} .
\end{aligned}
$$

Definition 2.2[2]. An algebra $(\mathcal{A}, \rightarrow, \sim,-, \sim$, 1) with a binary operations $" \rightarrow ", " \sim "$ and quasi complements " -", " " is called a pseudo-Wajsberg algebra if it satisfies the following axioms for all $x, y, z \in \mathcal{A}$,

(i) (a) $1 \rightarrow x=x$

(b) $1 \leadsto x=x$

(ii) $\quad(x \sim y) \rightarrow y=(y \sim x) \rightarrow x$ $=(y \rightarrow x) \backsim x=(x \rightarrow y) \backsim y$

(iii) (a) $(x \rightarrow y) \rightarrow((y \rightarrow z) \sim(x \rightarrow z))=1$

(b) $(x \sim y) \sim((y \sim z) \rightarrow(x \sim z))=1$

(iv) $\quad 1^{-}=1^{\sim}=0$

(v) (a) $\left(x^{-} \sim y^{-}\right) \rightarrow(y \rightarrow x)=1$

(b) $\left(x^{\sim} \rightarrow y^{\sim}\right) \sim(y \sim x)=1$

(vi) $\left(x \rightarrow y^{-}\right)^{\sim}=\left(y \sim x^{\sim}\right)^{-}$

Proposition 2.3[2]. A LPWA $(\mathcal{A}, \rightarrow, \sim,-\sim, 1)$ satisfies the following axioms for all $x, y \in \mathcal{A}$,

(i) $\quad x \rightarrow x=1, x \backsim x=1$

(ii) $\quad x \rightarrow(y \vee z)=(x \rightarrow y) \vee(x \rightarrow z)$

(iii) $\quad x \sim(y \vee z)=(x \sim y) \vee(x \sim z)$

(iv) $\quad(x \vee y) \rightarrow z=(x \rightarrow z) \wedge(x \rightarrow z)$

(v) $(x \vee y) \backsim z=(x \sim z) \wedge(x \sim z)$

(vi) $\quad x^{-} \rightarrow x=x ; x^{\sim} \sim x=x$

(vii) $\quad x \rightarrow x^{-}=x^{-} ; x \sim x^{\sim}=x^{\sim}$

(viii) $\left(x^{-} \sim 0\right) \rightarrow x=1 ;\left(x^{\sim} \rightarrow 0\right) \sim x=1$

(ix) $\quad x \rightarrow 0=x^{-} ; x \sim 0=x^{\sim}$

(x) $\quad 0 \rightarrow x=1 ; 0 \sim x=1$

(x) $\quad\left(x \rightarrow x^{-}\right)^{\sim}=x ;\left(x \sim x^{\sim}\right)^{-}=x$

(xi) $\quad\left(x^{\sim}\right)^{-}=\left(x^{-}\right)^{\sim}=x$

Definition 2.4[4]. Let $\mathcal{A}$ be LPWA. An intuitionistic fuzzy set $\quad T=\left(\mu_{T}, \gamma_{t}\right)$ of $\mathcal{A}$ is called an intuitionistic fuzzy implicative filter of $\mathcal{A}$ if it satisfies the following axioms for all $x, y \in \mathcal{A}$,

(i) $\quad \mu_{T}(1) \geq \mu_{T}(x) ; \gamma_{T}(1) \leq \gamma_{T}(x)$

(ii) $\quad \mu_{T}(y) \geq \min \left\{\mu_{T}(x \rightarrow y), \mu_{T}(x)\right\}$;

$\gamma_{T}(y) \leq \max \left\{\gamma_{T}(x \rightarrow y), \gamma_{T}(x)\right\}$

(iii) $\mu_{T}(y) \geq \min \left\{\mu_{T}(x \sim y), \mu_{T}(x)\right\}$; $\gamma_{T}(y) \leq \max \left\{\gamma_{T}(x \sim\right.$

$\left.y), \gamma_{T}(x)\right\}$ 
Definition 2.5[6]. Let $\mathcal{A}$ be LPWA. An intuitionistic fuzzy implicative filter $T=\left(\mu_{T}, \gamma_{T}\right)$ of $\mathcal{A}$ is called an intuitionistic fuzzy prime implicative filter of $\mathcal{A}$ if it satisfies the following axioms for all $x, y \in \mathcal{A}$,

(i) $\mu_{T}(x \vee y) \geq \min \left\{\mu_{T}(x), \mu_{T}(y)\right\}$

(ii) $\quad \gamma_{T}(x \vee y) \leq \max \left\{\gamma_{T}(x), \gamma_{T}(y)\right\}$

\section{MAIN RESULTS}

3.1. Intuitionistic Fuzzy Pseudo-Boolean implicative filter of Lattice Pseudo-Wajsberg Algebra

In this section, we define Intuitionistic fuzzy pseudo-Boolean implicative filter of LPWA, and obtain some results with illustrations.

Definition 3.1. Let $\mathcal{A}$ be LPWA. An intuitionistic fuzzy set $T=\left(\mu_{T}, \gamma_{T}\right)$ of $\mathcal{A}$ is called an intuitionistic fuzzy Pseudo-Boolean implicative filter of $\mathcal{A}$ if it satisfies the following axioms for all $x \in \mathcal{A}$,

(i) $\mu_{T}\left(x \vee x^{-}\right)=\mu_{T}(1) ; \gamma_{T}\left(x \vee x^{-}\right)=\gamma_{T}(1)$

(ii) $\mu_{T}\left(x \vee x^{\sim}\right)=\mu_{T}(1) ; \gamma_{T}\left(x \vee x^{\sim}\right)=\gamma_{T}(1)$

Example 3.2. Consider a set $\mathcal{A}=\{0, l, m, 1\}$. Define a partial ordering " $\leq$ " on $A$, such that $0 \leq l \leq m \leq 1$ and the binary operations " $\rightarrow$ "," $\sim$ " and quasi complements " -", " " given by the following tables 3.1., 3.2., 3.3., and 3.4.

\begin{tabular}{|c|c|}
\hline$x$ & $x^{-}$ \\
\hline 0 & 1 \\
\hline$l$ & $m$ \\
\hline$m$ & $l$ \\
\hline 1 & 0 \\
\hline
\end{tabular}

Table:3.1.

\section{Complement}

\begin{tabular}{|c|c|}
\hline$x$ & $x^{\sim}$ \\
\hline 0 & 1 \\
\hline$l$ & $m$ \\
\hline$m$ & $l$ \\
\hline 1 & 0 \\
\hline
\end{tabular}

Table:3.3. Complement

Consider an intuitionistic fuzzy subset $T=\left(\mu_{T}, \gamma_{T}\right)$ on $\mathcal{A}$ $\mu_{T}(x)=\left\{\begin{array}{lll}0.7 & \text { if } x \in\{0,1\} & \text { for all } x \in \mathcal{A} \\ 0.3 & \text { otherwise } & \text { for all } x \in \mathcal{A}\end{array}\right.$ $\gamma_{T}(x)=\left\{\begin{array}{lll}0.1 & \text { if } x \in\{0,1\} & \text { for all } x \in \mathcal{A} \\ 0.6 & \text { otherwise } & \text { for all } x \in \mathcal{A}\end{array}\right.$

Then $T$ is an intuitionistic fuzzy pseudo-Boolean implicative filter of $\mathcal{A}$.

Table:3.2.

Implication

\begin{tabular}{|c|c|c|c|c|}
\hline$\sim$ & 0 & $l$ & $m$ & 1 \\
\hline 0 & 1 & 1 & 1 & 1 \\
\hline$l$ & $m$ & 1 & 1 & 1 \\
\hline$m$ & $l$ & $l$ & 1 & 1 \\
\hline 1 & 0 & $l$ & $m$ & 1 \\
\hline
\end{tabular}

Table:3.4.

\section{Implication}

In the same Example 3.2, let us consider an intuitionistic fuzzy subset $T=\left(\mu_{T}, \gamma_{T}\right)$ on $\mathcal{A}$ as, $\mu_{T}(x)=$

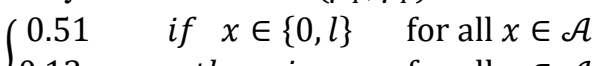
$\{0.13$ otherwise for all $x \in \mathcal{A}$

$\gamma_{T}(x)=\left\{\begin{array}{ccc}0.41 & \text { if } x \in\{0, l\} & \text { for all } x \in \mathcal{A} \\ 0.83 & \text { otherwise } & \text { for all } x \in \mathcal{A}\end{array}\right.$

Then $T$ is not an intuitionistic fuzzy pseudo-Boolean implicative filter of $\mathcal{A}$.

Since

$$
\begin{gathered}
\mu_{T}(l \vee m)=\mu_{T}((l \rightarrow m) \backsim m)=\mu_{T}((m \rightarrow l) \backsim l) \\
=\mu_{T}((l \backsim m) \rightarrow m)=\mu_{T}((m \sim l) \rightarrow l) \\
\mu_{T}(l \vee m)=\mu_{T}(l)=\mu_{T}(1)=\mu_{T}(m)=\mu_{T}(1) \\
\mu_{T}(l \vee m) \neq 0.5=0.2=0.2=0.2 \\
\gamma_{T}(l \vee m)=\gamma_{T}((l \rightarrow m) \backsim m)=\gamma_{T}((m \rightarrow l) \backsim l) \\
=\gamma_{T}((l \backsim m) \rightarrow m)=\gamma_{T}((m \sim l) \rightarrow l) \\
\gamma_{T}(l)=\gamma_{T}(1)=\gamma_{T}(m)=\gamma_{T}(1) \\
\gamma_{T}(l \vee m)=0.4 \neq 0.8=0.8=0.8
\end{gathered}
$$

Proposition 3.3. Let $T=\left(\mu_{T}, \gamma_{T}\right)$ be an intuitionistic fuzzy implicative filter of LPWA $\mathcal{A}$, then any intuitionistic fuzzy implicative filter of $\mathcal{A}$ is an intuitionistic fuzzy Pseudo-Boolean implicative filter of $\mathcal{A}$.

Proof. Let $T=\left(\mu_{T}, \gamma_{T}\right)$ be an intuitionistic fuzzy implicative filter of LPWA $\mathcal{A}$.

We have $\mu_{T}\left(x \vee x^{-}\right) \geq \min \left\{\mu_{T}\left(x \rightarrow\left(x \vee x^{-}\right)\right), \mu_{T}(x)\right\}$

$$
\begin{aligned}
& =\min \left\{\mu_{T}\left((x \rightarrow x) \vee\left(x \rightarrow x^{-}\right)\right), \mu_{T}(x)\right\} \\
& \quad[\text { From (ii) of proposition 2.3] } \\
& =\min \left\{\mu_{T}\left(1 \vee\left(x \rightarrow x^{-}\right)\right), \mu_{T}(x)\right\}
\end{aligned}
$$

$$
\begin{aligned}
& \text { [From (i) of proposition 2.3] } \\
\min \left\{\mu_{T}\left(x \rightarrow x^{-}\right), \mu_{T}(x)\right\} \leq \mu_{T}(1) &
\end{aligned}
$$

[From (i) of definition 2.4]

Thus $\mu_{T}\left(x \vee x^{-}\right)=\mu_{T}(1)$ for all $x \in \mathcal{A}$ and also

$$
\mu_{T}\left(x \vee x^{\sim}\right) \geq \min \left\{\mu_{T}\left(x \sim\left(x \vee x^{\sim}\right)\right), \mu_{T}(x)\right\}
$$

[From (iii) of definition 2.4]

$$
=\min \left\{\mu_{T}\left((x \sim x) \vee\left(x \sim x^{\sim}\right)\right), \mu_{T}(x)\right\}
$$

$$
=\min \left\{\mu_{T}\left(1 \vee\left(x \sim x^{\sim}\right)\right), \mu_{T}(x)\right\}
$$

[From (iii) of proposition 2.3]

$$
=\min \left\{\mu_{T}\left(x \sim x^{\sim}\right), \mu_{T}(x)\right\} \leq \mu_{T}(1)
$$

[From (i) of proposition 2.3]

[From (i) of definition 2.4]

Therefore, $\mu_{T}\left(x \vee x^{\sim}\right)=\mu_{T}(1)$ for all $x \in \mathcal{A}$

Similarly $\gamma_{T}\left(x \vee x^{-}\right) \leq \max \left\{\gamma_{T}\left(x \rightarrow\left(x \vee x^{-}\right)\right), \gamma_{T}(x)\right\}$

$$
=\max \left\{\gamma_{T}\left((x \rightarrow x) \vee\left(x \rightarrow x^{-}\right)\right), \gamma_{T}(x)\right\}
$$

[From (ii) of definition 2.4]

$$
=\max \left\{\gamma_{T}\left(1 \vee\left(x \rightarrow x^{-}\right)\right), \gamma_{T}(x)\right\}
$$

[From (ii) of proposition 2.3]

[From (i) of proposition 2.3]

$\gamma_{T}\left(x \vee x^{-}\right)=\max \left\{\gamma_{T}\left(x \rightarrow x^{-}\right), \gamma_{T}(x)\right\} \geq \gamma_{T}(1)$

for all $x \in \mathcal{A}$

[From (i) of definition 2.4] 
Therefore, $\gamma_{T}\left(x \vee x^{-}\right)=\gamma_{T}(1)$ and also

$$
\gamma_{T}\left(x \vee x^{\sim}\right) \leq \max \left\{\gamma_{T}\left(x \sim\left(x \vee x^{\sim}\right)\right), \gamma_{T}(x)\right\}
$$

[From (iii) of definition 2.4]

$$
=\max \left\{\gamma_{T}\left((x \sim x) \vee\left(x \sim x^{\sim}\right)\right), \gamma_{T}(x)\right\}
$$

[From (iii) of proposition 2.3]

$$
=\max \left\{\gamma_{T}\left(1 \vee\left(x \sim x^{\sim}\right)\right), \gamma_{T}(x)\right\}
$$

[From (i) of proposition 2.3]

$\gamma_{T}\left(x \vee x^{\sim}\right)=\max \left\{\gamma_{T}\left(x \sim x^{\sim}\right), \gamma_{T}(x)\right\} \geq \gamma_{T}(1)$

for all $x \in \mathcal{A}$

[From (i) of definition 2.4]

Thus, $\gamma_{T}\left(x \vee x^{\sim}\right)=\gamma_{T}(1)$ for all $x \in \mathcal{A}$

Hence, any intuitionistic fuzzy implicative filter of $\mathcal{A}$ is an intuitionistic fuzzy Pseudo-Boolean implicative filter of $\mathcal{A}$.

\section{Proposition 3.4}

Let $T=\left(\mu_{T}, \gamma_{T}\right)$ be an intuitionistic fuzzy Pseudo-Boolean implicative filter of LPWA $\mathcal{A}$, then which satisfies the following inequalities

(i) $\mu_{T}\left(x^{-}\right)=\mu_{T}\left(x \rightarrow x^{-}\right) ; \gamma_{T}\left(x^{-}\right)=\gamma_{T}\left(x \rightarrow x^{-}\right)$

(ii) $\mu_{T}\left(x^{\sim}\right)=\mu_{T}\left(x \sim x^{\sim}\right) ; \gamma_{T}\left(x^{\sim}\right)=\gamma_{T}\left(x \sim x^{\sim}\right)$ for all $x \in \mathcal{A}$.

Proof. Let $T=\left(\mu_{T}, \gamma_{T}\right)$ be an intuitionistic fuzzy Pseudo-Boolean implicative filter of LPWA $\mathcal{A}$.

$$
\text { Let } \mu_{T}\left(x^{-}\right)=\mu_{T}\left(1 \rightarrow x^{-}\right) \quad \text { [From (i)(a) of definition 2.2] }
$$$$
=\mu_{T}\left(1 \rightarrow\left(x \rightarrow x^{-}\right)\right)
$$

[From (vii) of proposition 2.3]

$$
=\mu_{T}\left(x \rightarrow x^{-}\right) \text {for all } x \in \mathcal{A}
$$

[From (i)(a) of definition 2.2]

$$
\begin{gathered}
\gamma_{T}\left(x^{-}\right)=\gamma_{T}\left(1 \rightarrow x^{-}\right) \quad \text { [From (i)(a) of definition 2.2] } \\
=\gamma_{T}\left(1 \rightarrow\left(x \rightarrow x^{-}\right)\right)
\end{gathered}
$$

[From (vii) of proposition 2.3] $=\gamma_{T}\left(x \rightarrow x^{-}\right)$for all $x \in \mathcal{A}$

[From (i)(a) of definition 2.2]

Similarly, we prove $\mu_{T}\left(x^{\sim}\right)=\mu_{T}\left(x \sim x^{\sim}\right)$ and $\gamma_{T}\left(x^{\sim}\right)=$ $\gamma_{T}\left(x \sim x^{\sim}\right)$ for all $x \in \mathcal{A}$.

Proposition 3.5. Let $T=\left(\mu_{T}, \gamma_{T}\right)$ be an intuitionistic fuzzy Pseudo-Boolean implicative filter of LPWA $\mathcal{A}$, then which satisfies the following inequalities

(i) $\mu_{T}\left(\left(x^{-}\right)^{\sim}\right)=\mu_{T}(x) ; \gamma_{T}\left(\left(x^{-}\right)^{\sim}\right)=\gamma_{T}(x)$

(ii) $\mu_{T}\left(\left(x^{\sim}\right)^{-}\right)=\mu_{T}(x) ; \gamma_{T}\left(\left(x^{\sim}\right)^{-}\right)=\gamma_{T}(x)$

for all $x \in \mathcal{A}$.

Proof. Let $T=\left(\mu_{T}, \gamma_{T}\right)$ be an intuitionistic fuzzy Pseudo-Boolean implicative filter of LPWA $\mathcal{A}$.

(i) Let $\mu_{T}(x)=\mu_{T}\left(x^{-} \rightarrow x\right)$ [From (vi) of proposition 2.3]

$$
=\mu_{T}\left(x^{-} \rightarrow\left(x^{\sim}\right)^{-}\right)
$$

[From (xi) of proposition 2.3]

$$
=\mu_{T}\left(x^{-} \rightarrow\left(x^{\sim} \rightarrow 0\right)\right)=\mu_{T}\left(x^{-} \sim 0\right)
$$

[From (ix) of proposition 2.3]

$=\mu_{T}\left(\left(x^{-}\right)^{\sim}\right)$ [From (ix) of proposition 2.3]

Similarly, we prove that $\gamma_{T}\left(\left(x^{-}\right)^{\sim}\right)=\gamma_{T}(x)$

(ii) Let $\mu_{T}(x)=\mu_{T}\left(x^{\sim} \sim x\right)$

[From (vi) of proposition 2.3]

$$
=\mu_{T}\left(x^{\sim} \rightarrow\left(x^{-}\right)^{\sim}\right)
$$

[From (xi) of proposition 2.3]

$$
=\mu_{T}\left(x^{\sim} \rightarrow\left(x^{-} \rightarrow 0\right)\right)=\mu_{T}\left(x^{\sim} \rightarrow 0\right)
$$

[From (ix) of proposition 2.3]

$$
=\mu_{T}\left(\left(x^{\sim}\right)^{-}\right)
$$

[From (ix) of proposition 2.3]

Similarly, we prove that $\gamma_{T}\left(\left(x^{\sim}\right)^{-}\right)=\gamma_{T}(x)$ for all $x \in \mathcal{A}$.

Proposition 3.6. Let $T=\left(\mu_{T}, \gamma_{T}\right)$ be an intuitionistic fuzzy Pseudo-Boolean implicative filter of LPWA $\mathcal{A}$. Let $T_{1}$ and $T_{2}$ be two intuitionistic fuzzy implicative filters of $\mathcal{A}, T_{1}$ is a subset of $T_{2}$ and $\mu_{T_{1}}(1)=\mu_{T_{2}}(1) ; \gamma_{T_{1}}(1)=\gamma_{T_{2}}$ (1). If $T_{1}$ is an intuitionistic fuzzy Pseudo-Boolean implicative filter of $\mathcal{A}$ then $T_{2}$ an intuitionistic fuzzy Pseudo-Boolean implicative filter $\mathcal{A}$.

Proof. Let $T_{1}$ be an intuitionistic fuzzy Pseudo-Boolean implicative filter of $\mathcal{A}$.

$\mu_{T_{1}}\left(x \vee x^{-}\right)=\mu_{T_{1}}(1) ; \gamma_{T_{1}}\left(x \vee x^{-}\right)=\gamma_{T_{1}}(1)$

and

$\mu_{T_{1}}\left(x \vee x^{\sim}\right)=\mu_{T_{1}}(1) ; \gamma_{T_{1}}\left(x \vee x^{\sim}\right)=\gamma_{T_{1}}(1)$

for all $x \in \mathcal{A}$.

And also $\mu_{T_{1}}\left(x \vee x^{-}\right) \leq \mu_{T_{2}}\left(x \vee x^{-}\right)$;

$$
\mu_{T_{1}}\left(x \vee x^{\sim}\right) \leq \mu_{T_{2}}\left(x \vee x^{\sim}\right)
$$

Since $T_{1}$ is a subset of $T_{2}$.

From the equation of (3.1) and $\mu_{T_{1}}(1)=\mu_{T_{2}}$ (1)

$\mu_{T_{2}}(1) \leq \mu_{T_{2}}\left(x \vee x^{-}\right) ; \mu_{T_{2}}(1) \leq \mu_{T_{2}}\left(x \vee x^{\sim}\right)$

From (i) and (ii) of definition 3.1.,

We have $\mu_{T_{2}}(1) \geq \mu_{T_{2}}\left(x \vee x^{-}\right) ; \mu_{T_{2}}(1) \geq \mu_{T_{2}}\left(x \vee x^{\sim}\right)$

From the equation of (3.2) and (3.3), we have

$\mu_{T_{2}}(1)=\mu_{T_{2}}\left(x \vee x^{-}\right) ; \mu_{T_{2}}(1)=\mu_{T}\left(x \vee x^{\sim}\right)$ for all $x \in \mathcal{A}$

Similarly $\gamma_{T_{1}}\left(x \vee x^{-}\right) \geq \gamma_{T_{2}}\left(x \vee x^{-}\right)$;

$$
\gamma_{T_{1}}\left(x \vee x^{\sim}\right) \geq \gamma_{T_{2}}\left(x \vee x^{\sim}\right)
$$

Since $T_{1}$ is a subset of $T_{2}$.

From the equation of (3.1) and $\gamma_{T_{1}}(1)=\gamma_{T_{2}}$ (1)

$\gamma_{T_{2}}(1) \geq \gamma_{T_{2}}\left(x \vee x^{-}\right) ; \gamma_{T_{2}}(1) \geq \gamma_{T_{2}}\left(x \vee x^{\sim}\right)$

From (ii) and (ii) of definition 3.1.,

We have $\gamma_{T_{2}}(1) \leq \gamma_{T_{2}}\left(x \vee x^{-}\right) ; \gamma_{T_{2}}(1) \leq \gamma_{T_{2}}\left(x \vee x^{\sim}\right)$

From the equation of (3.4) and (3.5), we have

$\gamma_{T_{2}}(1)=\gamma_{T_{2}}\left(x \vee x^{-}\right) ; \gamma_{T_{2}}(1)=\gamma_{T_{2}}\left(x \vee x^{\sim}\right) \quad$ for all $x \in$ $\mathcal{A}$.

Hence $T_{2}$ an intuitionistic fuzzy Pseudo-Boolean implicative filter of LPWA $\mathcal{A}$.

Proposition 3.7. Let $T=\left(\mu_{T}, \gamma_{T}\right)$ be an intuitionistic fuzzy Pseudo-Boolean implicative filter of LPWA $\mathcal{A}$. Let $T_{1}$ and $T_{2}$ be two intuitionistic fuzzy Pseudo-Boolean implicative filters of $\mathcal{A}$. Then intersection of $T_{1}$ and $T_{2}$ is also an intuitionistic fuzzy Pseudo-Boolean implicative filter of $\mathcal{A}$.

Proof. Let $T_{1}$ and $T_{2}$ be an intuitionistic fuzzy Pseudo-Boolean implicative filter of LPWA $\mathcal{A}$.

Then , we have

$$
\begin{aligned}
& \mu_{T_{1}}\left(x \vee x^{-}\right)=\mu_{T_{1}}(1) ; \gamma_{T_{1}}\left(x \vee x^{-}\right)=\gamma_{T_{1}}(1) \\
& \mu_{T_{1}}\left(x \vee x^{\sim}\right)=\mu_{T_{1}}(1) ; \gamma_{T_{1}}\left(x \vee x^{\sim}\right)=\gamma_{T_{1}}(1) \quad \text { and } \\
& \mu_{T_{2}}\left(x \vee x^{-}\right)=\mu_{T_{2}}(1) ; \gamma_{T_{2}}\left(x \vee x^{-}\right)=\gamma_{T_{2}}(1) \\
& \mu_{T_{2}}\left(x \vee x^{\sim}\right)=
\end{aligned}
$$

$\mu_{T_{2}}(1) ; \gamma_{T_{2}}\left(x \vee x^{\sim}\right)=\gamma_{T_{2}}(1)$ 
for all $x \in \mathcal{A}$.

Let $\quad \mu_{\left(T_{1} \cap T_{2}\right)}\left(x \vee x^{-}\right)=\mu_{T_{1}}\left(x \vee x^{-}\right) \wedge \mu_{T_{2}}\left(x \vee x^{-}\right)$

$$
=\mu_{\left(T_{1} \cap T_{2}\right)}(1) \text { for all } x \in \mathcal{A} \text {. }
$$

Similarly, we prove $\gamma_{\left(T_{1} \cap T_{2}\right)}\left(x \vee x^{-}\right)=$

$\gamma_{\left(T_{1} \cap T_{2}\right)}(1)$ for all $x \in \mathcal{A}$.

Hence, intersection of $T_{1}$ and $T_{2}$ is also an intuitionistic fuzzy Pseudo-Boolean implicative filter of $\mathcal{A}$.

Proposition 3.8. Let $T=\left(\mu_{T}, \gamma_{T}\right)$ be an intuitionistic fuzzy Pseudo-Boolean implicative filter of LPWA $\mathcal{A}$, then which satisfies the following inequalities

(i) $\mu_{T}(1)=\mu_{T}(x)$ (or) $\mu_{T}(1)=\mu_{T}\left(x^{-}\right)$;

$\gamma_{T}(1)=\gamma_{T}(x)$ (or) $\gamma_{T}(1)=\gamma_{T}\left(x^{-}\right)$

(ii) $\mu_{T}(1)=\mu_{T}$ (x) (or) $\mu_{T}$ (1) $=\mu_{T}\left(x^{\sim}\right)$;

$\gamma_{T}(1)=\gamma_{T}(x)$ (or) $\gamma_{T}(1)=\gamma_{T}\left(x^{\sim}\right)$ for all $x \in \mathcal{A}$.

Proof. Let $T=\left(\mu_{T}, \gamma_{T}\right)$ be an intuitionistic fuzzy Pseudo-Boolean implicative filter of LPWA $\mathcal{A}$.

So $\mu_{T}(1)=\mu_{T}\left(x \vee x^{-}\right)$

$\geq \min \left\{\mu_{T}\left(x^{-}\right), \mu_{T}(x)\right\}$ [From (i) of definition 2.4]

$=\mu_{T}(x)$ (or) $\mu_{T}\left(x^{-}\right)$

$\mu_{T}(1)=\mu_{T}(x)$ (or) $\mu_{T}(1)=\mu_{T}\left(x^{-}\right)$and also

$\gamma_{T}(1)=\gamma_{T}\left(x \vee x^{-}\right)$

$\leq \max \left\{\gamma_{T}\left(x^{-}\right), \gamma_{T}(x)\right\}$

$=\gamma_{T}(x)$ (or) $\gamma_{T}\left(x^{-}\right) \quad$ [From (ii) of definition 2.4]

$\gamma_{T}(1)=\gamma_{T}(x)$ (or) $\gamma_{T}(1)=\gamma_{T}\left(x^{-}\right)$

Similarly, we prove

$\mu_{T}(1)=\mu_{T}(x) \quad($ or $) \mu_{T}(1)=\mu_{T}\left(x^{\sim}\right) ; \gamma_{T}(1)=\gamma_{T}(x)$

(or) $\gamma_{T}(1)=\gamma_{T}\left(x^{\sim}\right)$ for all $x \in \mathcal{A}$.

Proposition 3.9. Let $T=\left(\mu_{T}, \gamma_{T}\right)$ be an intuitionistic fuzzy Pseudo-Boolean implicative filter of LPWA $\mathcal{A}$, then which satisfies the following

(i) $\mu_{T}(1)=\mu_{T}(x)$ (or) $\mu_{T}(1)=\mu_{T}\left(x^{-}\right)$if and only if $\mu_{T}\left(x \rightarrow x^{-}\right)=\mu_{T}$ (1) (or) $\mu_{T}\left(x^{-} \rightarrow x\right)=\mu_{T}$ (1)

(ii) $\gamma_{T}(1)=\gamma_{T}(x)$ (or) $\gamma_{T}(1)=\gamma_{T}\left(x^{-}\right)$if and only if $\gamma_{T}\left(x \rightarrow x^{-}\right)=\gamma_{T}(1)$ (or) $\gamma_{T}\left(x^{-} \rightarrow x\right)=\gamma_{T}(1)$

(iii) $\mu_{T}(1)=\mu_{T}(x)$ (or) $\mu_{T}(1)=\mu_{T}\left(x^{\sim}\right)$ if and only if $\mu_{T}\left(x \rightarrow x^{\sim}\right)=\mu_{T}$ (1) (or) $\mu_{T}\left(x^{\sim} \rightarrow x\right)=\mu_{T}$ (1)

(iv) $\gamma_{T}(1)=\gamma_{T}(x) \quad$ (or) $\quad \gamma_{T}(1)=\gamma_{T}\left(x^{\sim}\right)$ if and only if $\gamma_{T}\left(x \rightarrow x^{\sim}\right)=\gamma_{T}(1)($ or $) \quad \gamma_{T}\left(x^{\sim} \rightarrow x\right)=\gamma_{T}(1)$

for all $x \in \mathcal{A}$.

Proof. Let $T=\left(\mu_{T}, \gamma_{T}\right)$ be an intuitionistic fuzzy Pseudo-Boolean implicative filter of LPWA $\mathcal{A}$.

(i) Let $\mu_{T}(1)=\mu_{T}(x)$ (or) $\mu_{T}(1)=\mu_{T}\left(x^{-}\right)$

To Prove : $\mu_{T}\left(x \rightarrow x^{-}\right)=\mu_{T}$ (1) (or)

$$
\mu_{T}\left(x^{-} \rightarrow x\right)=\mu_{T}(1)
$$

From (i) of definition 3.1, we have

$$
\mu_{T}\left(x \rightarrow x^{-}\right) \vee \mu_{T}\left(x^{-} \rightarrow x\right)=\mu_{T}(1)
$$

From (i) the proposition 3.8, we have

$$
\mu_{T}\left(x \rightarrow x^{-}\right)=\mu_{T}(1) \text { (or) } \mu_{T}\left(x^{-} \rightarrow x\right)=\mu_{T}(1)
$$

Conversely,

let $\mu_{T}\left(x \rightarrow x^{-}\right)=\mu_{T}$ (1) (or) $\mu_{T}\left(x^{-} \rightarrow x\right)=\mu_{T}$ (1)

and $\mu_{T}\left(x \vee x^{-}\right)=\mu_{T}(1)$

Now $\left(x^{-} \vee x\right) \rightarrow x=\left(x^{-} \rightarrow x\right) \wedge(x \rightarrow x)$

[From (iv) of proposition 2.3]

$$
=\left(x^{-} \rightarrow x\right) \wedge 1=\left(x^{-} \rightarrow x\right)
$$

[From (i) of proposition 2.3]

It follows that $\mu_{T}\left(\left(x^{-} \vee x\right) \rightarrow x\right)=\mu_{T}\left(x^{-} \rightarrow x\right)=\mu_{T}(1)$ Since, $\mu_{T}(x) \geq \min \left\{\mu_{T}\left(\left(x^{-} \vee x\right) \rightarrow x\right), \mu_{T}\left(x^{-} \vee x\right)\right\}$

[From (ii) of definition 2.4]

So, $\mu_{T}(x)=\mu_{T}(1)$

Now $\left(x \vee x^{-}\right) \rightarrow x^{-}=\left(x \rightarrow x^{-}\right) \wedge\left(x^{-} \rightarrow x^{-}\right)$

[From (iv) of proposition 2.3]

$$
=\left(x \rightarrow x^{-}\right) \wedge 1=\left(x \rightarrow x^{-}\right)
$$

[From (i) of proposition 2.3]

It follows that $\mu_{T}\left(\left(x \vee x^{-}\right) \rightarrow x^{-}\right)=\mu_{T}\left(x \rightarrow x^{-}\right)=\mu_{T}(1)$ Since, $\mu_{T}\left(x^{-}\right) \geq \min \left\{\mu_{T}\left(\left(x \vee x^{-}\right) \rightarrow x\right), \mu_{T}\left(x \vee x^{-}\right)\right\}$

[From (ii) of definition 2.4]

Thus, $\mu_{T}\left(x^{-}\right)=\mu_{T}(1)$.

(ii) Assume that $\gamma_{T}(1)=\gamma_{T}(x)$ (or) $\gamma_{T}(1)=\gamma_{T}\left(x^{-}\right)$

Prove that $\gamma_{T}\left(x \rightarrow x^{-}\right)=\gamma_{T}$ (1) (or) $\gamma_{T}\left(x^{-} \rightarrow x\right)=\gamma_{T}(1)$

From (i) of definition 3.1,

We have $\gamma_{T}\left(x \rightarrow x^{-}\right) \vee \gamma_{T}\left(x^{-} \rightarrow x\right)=\gamma_{T}(1)$

From (i) the proposition 3.8, we have

$$
\gamma_{T}\left(x \rightarrow x^{-}\right)=\gamma_{T}(1)(\text { or }) \gamma_{T}\left(x^{-} \rightarrow x\right)=\gamma_{T}(1)
$$

Conversely,

Let $\gamma_{T}\left(x \rightarrow x^{-}\right)=\gamma_{T}$ (1) (or) $\gamma_{T}\left(x^{-} \rightarrow x\right)=\gamma_{T}(1) \quad$ and $\gamma_{T}\left(x \vee x^{-}\right)=\gamma_{T}(1)$ and

$\left(x^{-} \vee x\right) \rightarrow x=\left(x^{-} \rightarrow x\right) \wedge(x \rightarrow x)$

[From (iv) of proposition 2.3]

$$
=\left(x^{-} \rightarrow x\right) \wedge 1=\left(x^{-} \rightarrow x\right)
$$

[From (i) of proposition 2.3]

It follows that $\gamma_{T}\left(\left(x^{-} \vee x\right) \rightarrow x\right)=\gamma_{T}\left(x^{-} \rightarrow x\right)=\gamma_{T}(1)$

Since $\gamma_{T}(x) \leq \max \left\{\gamma_{T}\left(\left(x^{-} \vee x\right) \rightarrow x\right), \gamma_{T}\left(x^{-} \vee x\right)\right\}$

[From (ii) of definition 2.4]

Thus, $\gamma_{T}(x)=\gamma_{T}(1)$

and $\left(x \vee x^{-}\right) \rightarrow x^{-}=\left(x \rightarrow x^{-}\right) \wedge\left(x^{-} \rightarrow x^{-}\right)$

[From (iv) of proposition 2.3]

$$
=\left(x \rightarrow x^{-}\right) \wedge 1=\left(x \rightarrow x^{-}\right)
$$

[From (i) of proposition 2.3]

It follows that $\gamma_{T}\left(\left(x \vee x^{-}\right) \rightarrow x^{-}\right)=\gamma_{T}\left(x \rightarrow x^{-}\right)=\gamma_{T}(1)$

Since $\gamma_{T}\left(x^{-}\right) \leq \max \left\{\gamma_{T}\left(\left(x \vee x^{-}\right) \rightarrow x\right), \gamma_{T}\left(x \vee x^{-}\right)\right\}$

[From (ii) of definition 2.4]

So, $\gamma_{T}\left(x^{-}\right)=\gamma_{T}(1)$

Similarly, we prove that (iii) and (iv).

\section{CONCLUSION}

In this paper, we have introduced the notion of an intuitionistic fuzzy pseudo-Boolean implicative filter of LPWA and discussed some properties with illustrations.

\section{REFERENCES}

1. Atanassov, K.T., Intuitionistic fuzzy sets, Fuzzy Sets and Systems, 20(1) (1986) 87-96.

2. Ceterchi Rodica, The Lattice Structure of Pseudo-Wajsberg Algebras, Journal of universal Computer Science, 6 (2000), 22-38.

3. Font, J. M., Rodriguez, A. J., and Torrens, A., Wajsberg algebras, Stochastica, 8 (1984) 5-31.

\section{Published By:}

Blue Eyes Intelligence Engineering \& Sciences Publication 
4. Ibrahim, A., and Jeya Lekshmi, K., Intuitionistic Fuzzy Implicative Filters of lattice Pseudo-Wajsberg Algebras, Journal of Applied Science and Computations, 5 (2018), 327-336

5. Ibrahim, A., and Jeya Lekshmi, K., Pseudo-Boolean and Fuzzy Pseudo-Boolean Implicative Filters of Lattice Pseudo-Wajsberg Algebras, Advances in Mathematics; Scientific Journal 8, 3(2019), 311-320.

6. Ibrahim, A., and Jeya Lekshmi, K., Branches of Prime Implicative Filters of Lattice Pseudo-Wajsberg Algebras, (communicated).

7. Wajsberg, M., Beiträge zum Metaaussagenkalkül I, Monat. Mat. Phys. 42, (1935), 221-242.

8. Zadeh, L. A., Fuzzy sets, Information Control 8 (1965), 338-353.

\section{AUTHORS PROFILE}

Dr. A. Ibrahim is working as Assistant Professor in $\mathrm{P}$ .G. and Research Department of Mathematics, H. H. The Rajah's College, Pudukkottai, Affiliated to Bharathidasan University, Tiruchirappalli, Tamilnadu,India. He has worked as Head of the department in Mathematics, Texcity Arts and Science College, Coimbatore, Tamilnadu, India. He has more than 22 years teaching experience at collegiate level. His research areas are fuzzy Mathematics and theoretical Computer Science etc.

K. Jeya Lekshmi received her B.Sc in Mathematics from Sree Ayyappa College for women, Chunkankadai, Kanayakumari District, TamilNadu, India. M.sc from Sivanthi Aditanar College, Nagercoil, Kanayakumari District, TamilNadu, India and M.Phil in Madurai Kamaraj University. Presently, She is Pursuing part time Ph.D under guidance of Dr. A .Ibrahim. Her research area is Fuzzy Algebra. She is working as Assistant Professor in Rathnavel Subramaniam College of Arts and Science, Sulur, Coimbatore,Tamil Nadu \& Research Scholar, P.G. and Research Department of Mathematics, H. H. The Rajah's College, Pudukkottai, Affiliated to Bharathidasan University, Tiruchirappalli,, Tamilnadu, India 